\title{
Talleres de creatividad en islas virtuales: espacios participativos en línea
}

\section{Creativity workshops in virtual islands: participatory online spaces}

\author{
DAVID SERRA \\ Departamento de Filología y Comunicación. Universidad de Girona. \\ david.serranavarro@udg.edu
}

Recibido: 15 de febrero de 2012

Aprobado: 27 de junio de 2012

\begin{abstract}
Resumen
La educación artística, en combinación con las nuevas tecnologías, ha encontrado una nueva fuente de recursos en la que desarrollarse y expandirse. En estas nuevas relaciones, donde las técnicas tradicionales se funden con los sistemas digitales, nos centraremos en una de las prácticas colaborativas realizadas en el marco europeo de Villes 3.0. En concreto en un taller de creatividad basado en las interrelaciones que pueden generarse entre los mundos virtuales y la expresión plástica, dando visibilidad al potencial pedagógico que nos ofrecen los nuevos espacios de realidad virtual en línea.
\end{abstract}

Palabras clave: taller creatividad, mundo virtual, interactividad, educación artística.

Serra, D. (2013): Talleres de creatividad en islas virtuales: espacios participativos en línea. Arte, Individuo y Sociedad, 25(2) 179-188

\begin{abstract}
Art education, combined with new technologies, has found a new source of resources to grow and expand. In these new relationships, where traditional techniques are fused with digital systems, we focus on a collaborative practices carried out under the european program Villes 3.0. Specifically in a creativity workshop based on the interrelationships that may arise between virtual worlds and artistic expression, giving visibility to the educational potential offered us by the new spaces of virtual reality online.
\end{abstract}

Key Words: creativity workshop, virtual world, interactivity, art education.

Serra, D. (2013): Creativity workshops in virtual islands: participatory online spaces. Arte, Individuo y Sociedad, 25(2) 179-188

Sumario: 1. Introducción, 2. Plataforma de acción: Second Life, 2.1. Islas virtuales, 3. Praxis en tiempo real, 3.1. Creación plástica a través de un avatar, 3.2. Objeto final: Machinima, 3.3. Evaluación, 4. Culdesac Island laboratorio plural. Referencias. 


\section{Introducción}

Durante un período de tres años (2009-2011) ha tenido lugar el programa de cooperación territorial Ciudades 3. $0^{1}$, financiado por los fondos europeos INTERREG y cuyo escenario reunía en un proyecto común las ciudades de Huesca (Aragón), Olot (Cataluña) y Tournefeuille (Midi-Pyrénées). El objetivo de esta alianza cultural era doble: reforzar el papel de polos culturales descentralizados, y crear una relación de proximidad y de complicidad entre el arte y los ciudadanos, especialmente ocupando el espacio público. En este marco transfronterizo se inscribieron una serie de actividades artísticas, entre las cuales destacaremos el laboratorio virtual Culdesac Island $^{2}$.

La idea principal de esta propuesta, dirigida por el artista Kenneth Russo, consistía en acercar a la ciudadanía nuevas formas de interacción social en red, promover la colaboración desterritorializada, dar a conocer la noción de mundos virtuales y estimular mediante talleres semipresenciales nuevos procesos creativos en las praxis artísticas contemporáneas. A lo largo de este singular proyecto, en un contexto de corte intergeneracional, se materializaron un conjunto de experiencias pedagógicas y artísticas que tomaremos como estrategias didácticas a través de la formación y reflexión crítica de los entornos virtuales.

\section{Plataforma de acción: Second Life}

Culdesac Island se estableció como un laboratorio social, un banco de pruebas experimentales de acceso abierto y un espacio de encuentro. No obstante, antes de describir la metodología de las acciones desarrolladas, nos es de fundamental importancia señalar los recursos tecnológicos utilizados; en concreto, determinar qué motivos propiciaron la elección de la plataforma virtual Second Life $e^{3}$.

Los mundos virtuales suelen caracterizarse por su persistencia, interactividad y corporeidad (Castronova, 2001), tres factores clave que se repiten en plataformas como There, Entropia Univers, Habbo Hotel, Gaia Online, etc. Sin embargo, Second Life reúne un conjunto de singularidades que lo convierten en modelo único respecto a otras posibles plataformas análogas. El metaverso ${ }^{4}$ de Second Life se constituye como un universo paralelo en el que los usuarios no están subordinados a ningún objetivo concreto, simplemente interactuar. No se trata de un simple chat 3D, sino de un mundo que proporciona las herramientas necesarias para la libre creación virtual. La economía ${ }^{5}$ de este entorno estimula la creación de contenidos, ya que el tránsito de objetos creados configuran la base de intercambio cultural y transacción económica. Es decir, cada creación tiene un valor económico real, hecho que explica el interés de la compañía para favorecer al máximo los recursos y garantías en la generación de contenidos. Second Life se construye mediante código abierto y protege los derechos de autor bajo licencias Creative Commons, dos razones más para entender una actividad artística libre y sin restricciones de autoría. Cabe señalar también que su ejecutable funciona sobre varias plataformas, y que contempla dos modalidades de uso: el Teen Grid , pensado para menores y bajo la supervisión de un tutor; y su versión homóloga para adultos. 


\subsection{Islas virtuales}

El espacio del metaverso está segmentado y articulado mediante islas virtuales, talmente como nodos Peer-to-peer $(p 2 p)^{7}$, autónomos e interconectados permanentemente. Comparativamente podemos asociar la geografía virtual como un gran hipertexto en el que cada isla cumple la función de lexia, de bloque de significación. Roland Barthes en $S / Z$ entiende la lexia como "una serie de cortos fragmentos contiguos que constituyen unidades de lectura" (1970: 18), de tal forma que cada isla se comporta como un marco de significación intersubjetiva. En paralelo, la idea de isla conlleva una cierta connotación de aislamiento y singularidad, remitiéndonos a una "representación-metáfora" en la que sus "usuarios-habitantes" agudizan su inmersión simbólica. La insularidad de Second Life nos delimita un conjunto de submundos que funcionan simultáneamente, conectados, pero a su a vez con distintos pactos simbólicos. Una estructura en la que los avatares se ven obligados a actualizarse, y la exploración va más allá de la "imagen-forma"; es decir, en cada isla se diversifica la experiencia y se amplía el conocimiento mediante un proceso de comunicación interactiva con el entorno virtual, a su vez social.

\section{Praxis en tiempo real}

Si lo virtual deviene otro estado de la realidad, no material y no irreal, uno de los ejes que marcó los distintos talleres fue la noción "en tiempo real". Partiendo de esta idea, la investigación-formación no suponía trabajar en diferido, sino visualizar los resultados de forma inmediata y pública. Dada la diversidad de intervenciones que se dieron durante varios meses, clasificaremos brevemente algunas de las actividades artísticas más tipificadas de los mundos virtuales con la intención de contextualizar la sesiones realizadas entre participantes y artista: machinima $^{8}$, code-performance ${ }^{9} \mathrm{e}$ hyperformalismo ${ }^{10}$.

- Machinima: es un término que se refiere a la creación de piezas audiovisuales utilizando los gráficos de los videojuegos como elementos articuladores de una narración. Una relación entre los conceptos machine y cinema (Kelland y Dave, 2005).

- Code-performance: hace referencia al género artístico de la performance, pero esta vez trasladado en el espacio virtual y actuado mediante un nuevo cuerpo que encarna el avatar.

- Hyperformalismo: se relaciona con todas aquellos objetos virtuales creados desde la visión de un espacio generado matemáticamente. El objeto como algoritmo.

Estos tres bloques nos delimitan las líneas de trabajo en que los usuarios iniciaron su producción colaborativa, un recorrido por la posibilidades hipertextuales en donde se combinaban nuevas identidades digitales y nuevos canales de audiencia. De esta manera, pondremos de relieve un taller enfocado únicamente a los niños: "Creación plástica a través de un avatar". 


\subsection{Creación plástica a través de un avatar}

Edad de los participantes: niños de 7 años a 10 años.

Número de participantes: 9 alumnos.

Lugar: Escuela Pigment (Olot)

Tutor supervisor de la actividad: Marian Vayreda

Sesiones: cuatro sesiones de 2 horas.

Metodología y contenido:

Se inicia el taller visualizando un entorno virtual con un proyector, a continuación se realiza una exploración visual del mundo virtual guiándonos por las sugerencias de los participantes. Se entra en el juego de construcción de experiencia 3D a modo de "psycogeografia", talmente como conocer el mundo bajo la óptica de los mapas de Guy Debord. Una vez contextualizado el marco de actuación, se muestra la posibilidad de transferir contenidos analógicos (dibujos, sonido, esculturas) a contenidos digitales-virtuales. Esta prueba empírica es el punto de salida para que los participantes empiecen a negociar y diseñar el tipo de intervención plástica que desean transformar en virtual. Un proceso de autoaprendizaje donde el escenario virtual entra en diálogo con las actitudes y aptitudes creativas.

\section{Sesión 1: Dérive en el entorno 3D}

Sin un rumbo concreto, el tutor se desplaza de forma azarosa por los espacios 3D de la plataforma Second Life, recibiendo instrucciones de los alumnos para tomar una dirección concreta en función de los tres ejes cartesianos, los cuales interiorizan los asistentes como referentes de posicionamiento espacial. Este proceso consiste en una identificación del medio en la modalidad más mimética de entendimiento del espacio 3D, y sus formas arquitectónicas (Fig.1).

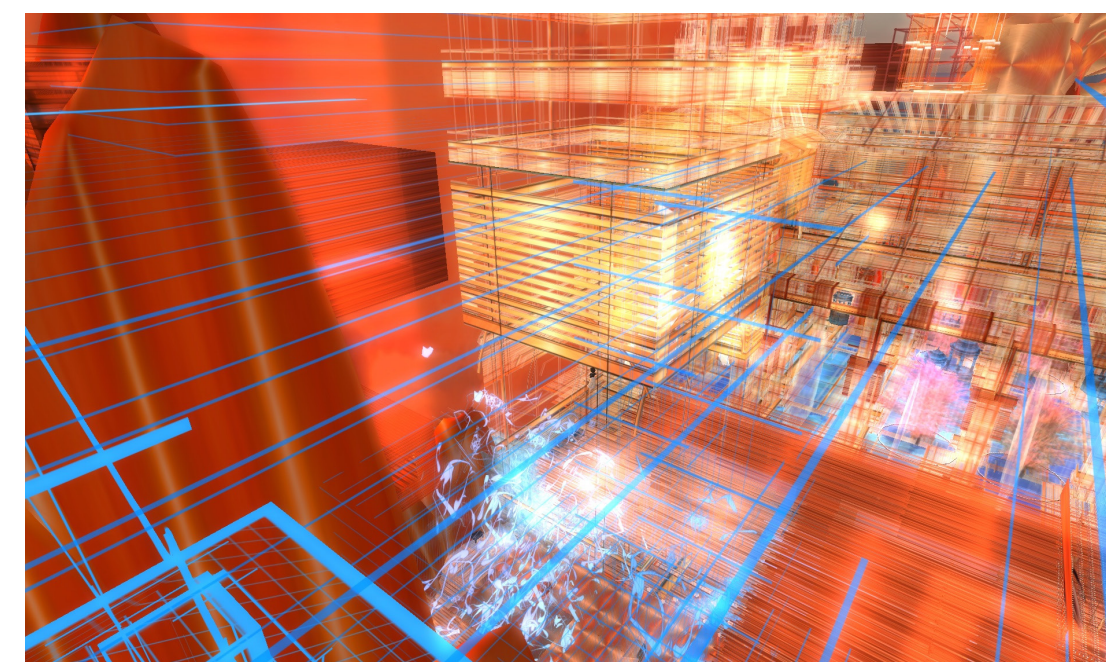

Figura 1. Captura de espacio arquitectónico de la sesión 1. Autor: Kenneth Russo. 


\section{Sesión 2: Transferencia de contenidos}

Una vez explorado el universo paralelo bajo una visión subjetiva en primera persona, se procede a mostrar el espacio en relación al personaje virtual que lo recorre. Este paso supone el cambio a una concepción del medio en tercera persona, fijándose el avatar como signo identadario que encabeza la experiencia. Precisamente, este alter-ego es el objeto donde se focalizará el taller, y se usará como ejemplo de transferencia entre lo real y lo virtual. Basándonos en el mapeo de superfíces de los volúmenes, se realiza un diseño gráfico en base una plantilla determinada, se escanea, y se transfiere sobre el objeto virtual (Fig.2 y Fig.3).
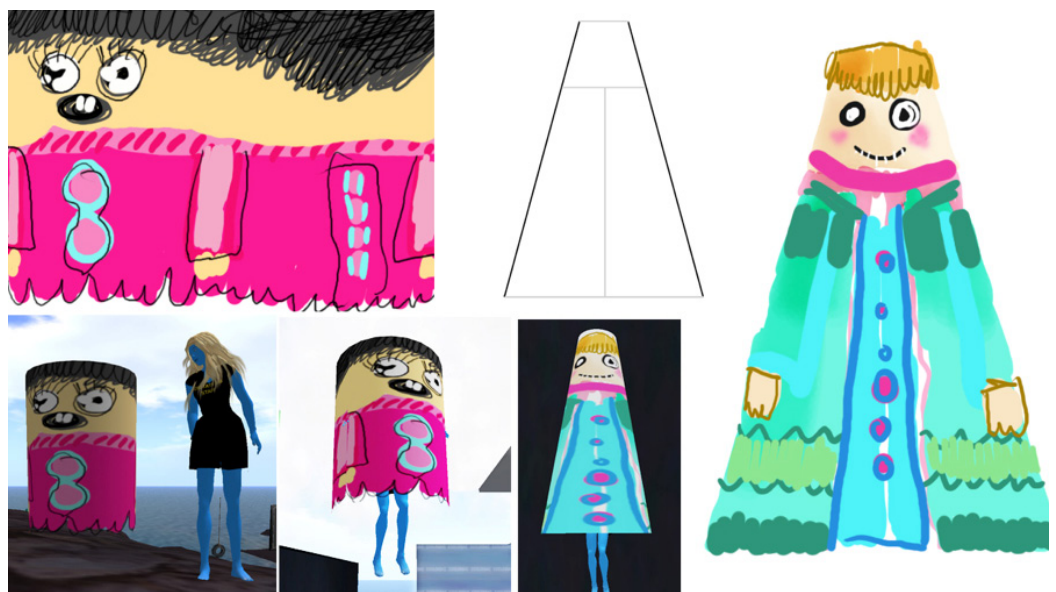

Figura 2. 2D sobre papel, escaneado, y resultado del mapeado sobre volumen virtual. Autor: Kenneth Russo.

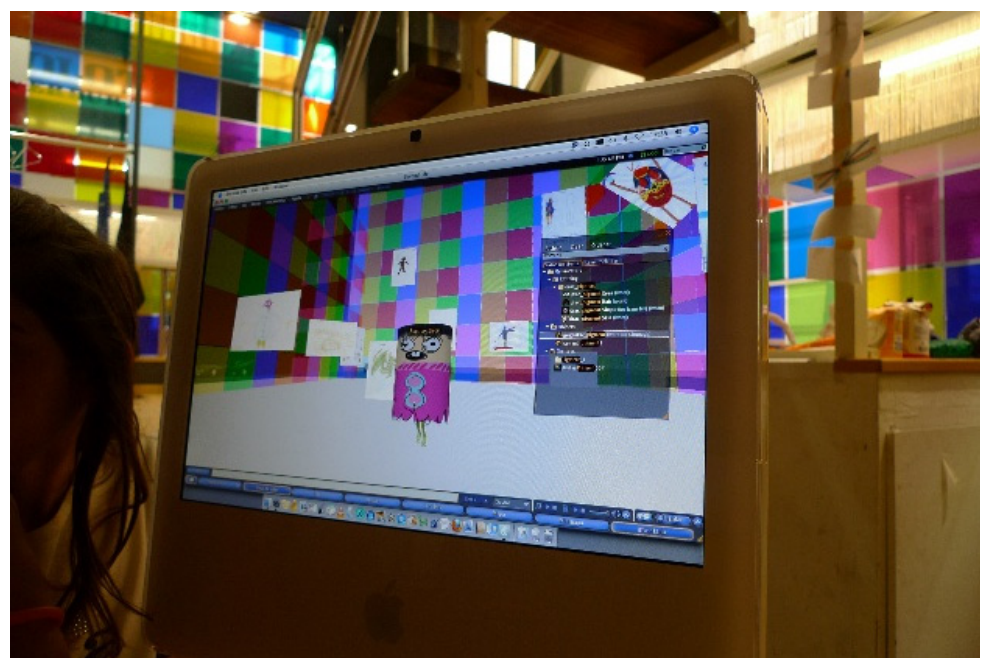

Figura 3. Visualización, en monitor de ordenador en línea, de los resultados en aula virtual. Escuela Pigment, Olot. Autor: Kenneth Russo. 


\section{Sesión 3: Diseño y elaboración de un alter-ego}

En esta fase los alumnos trabajan de forma individual en la construcción 2D de una futura identidad digital. Mediante distintos materiales y utensilios, efectúan un conjunto de dibujos que tendrán como función su proyección virtual en forma de personaje 3D. Dado que lo interesante es ver los resultados de forma inmediata, la estrategia consiste en generar aproximaciones gráficas para comprobar a través del método ensayo y error si la transferencia realizada encaja con sus expectativas imaginadas (Fig. 4). De esta manera cada participante acumula un pequeño inventario virtual de diseños que posteriormente serán expuestos.
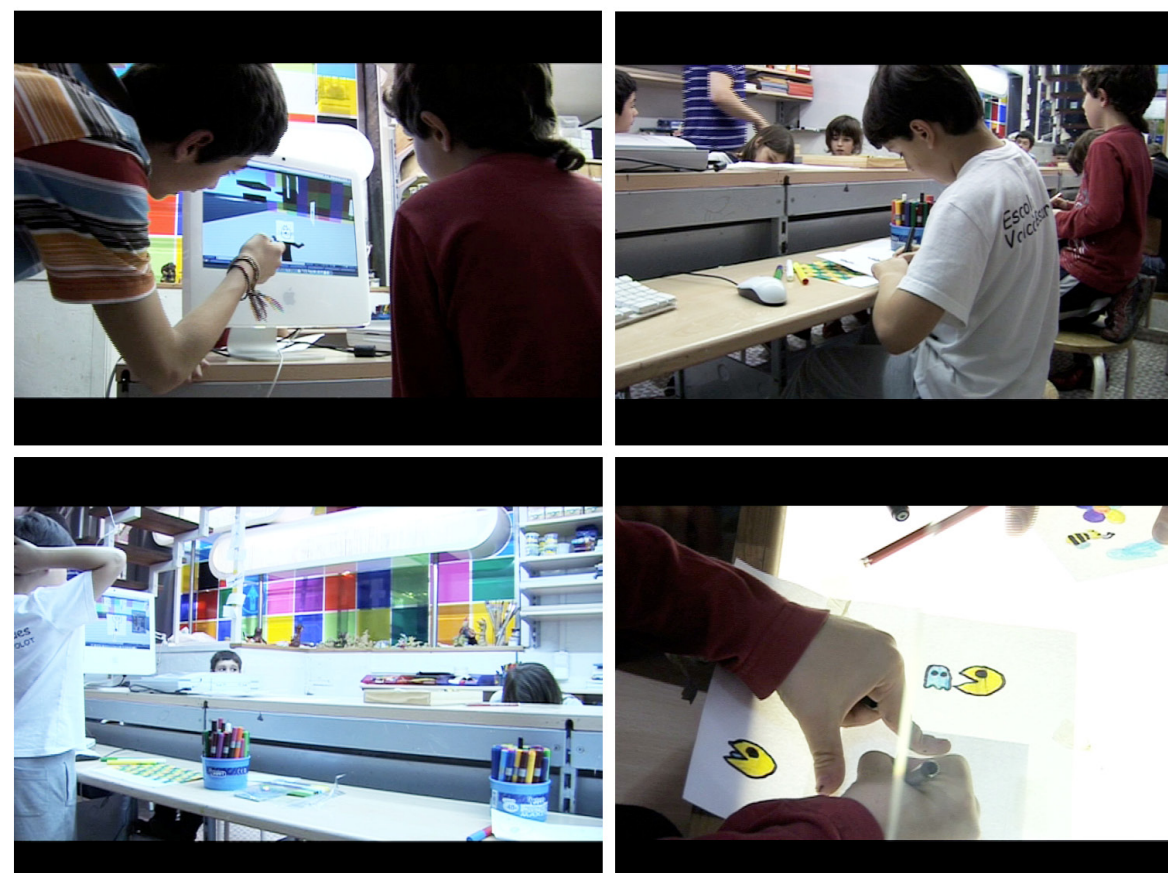

Figura 4. Diferentes fases del proceso creativo realizado por los alumnos de la Escuela Pigment. Autor: Kenneth Russo.

\section{Sesión 4: El museo interactivo}

En esta última sesión, bajo la supervisión del tutor, se procede a la selección final de trabajos que tendrán una doble finalidad: una exposición gráfica en la aula virtual (Fig. 5), y un dispensador virtual de diseños públicos. Es decir, cualquier avatar que visite la exposición podrá utilizar los diseños para su propia representación. Esta característica es fundamental, ya que se está recreando una dinámica de trabajo basada en la arquitectura colaborativa y en procesos de coautoría. Simultáneamente cada participante es informado de sus derechos Creative Commons, con lo que decidirá libremente si acepta que otro sujeto pueda utilizar, copiar y/o modificar su creación. 


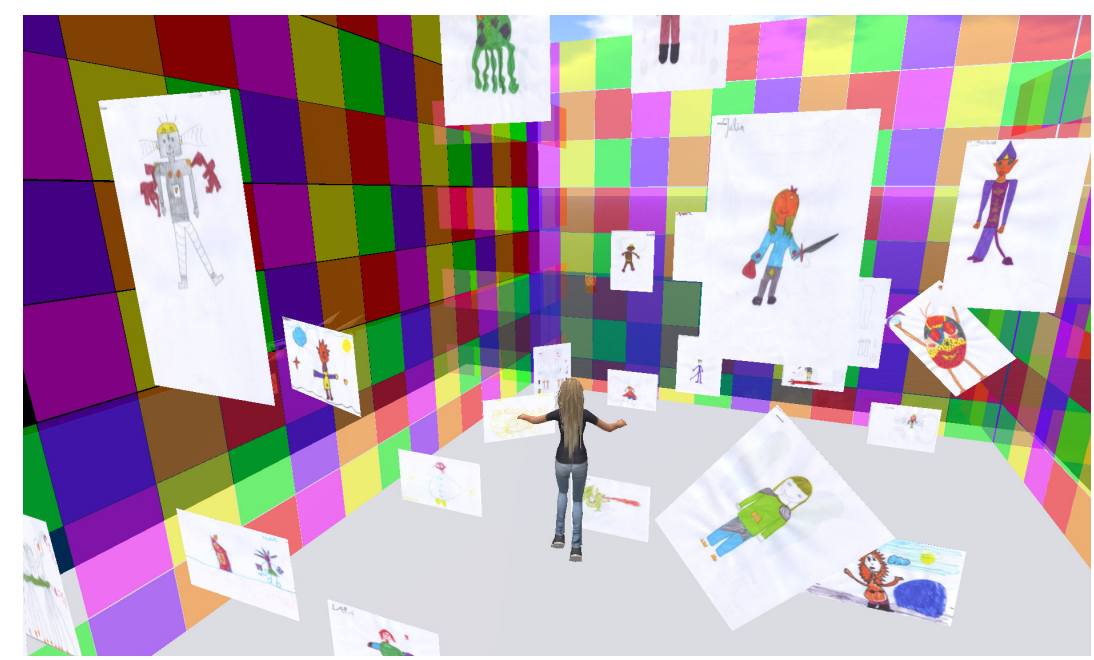

Figura 5. Captura de la interfaz mientras se desarrolla una visita, en tiempo real, en el espacio espositivo 3D. Autor: Kenneth Russo.

\subsection{Objeto final: Machinima}

Paralelamente, desde la primera sesión, todas las acciones visualizadas en el monitor han sido registradas en formato video. Transcurridos unos días, mediante este material audiovisual, y después de una previa posproducción realizada por el artista, se entrega una copia a cada uno de los participantes. Este obsequio machinima permite una sesión extraordinaria en la que se comenta el desarrollo general del taller. Esencialmente lo que se pretende es recuperar y documentar la experiencia de un entorno efímero, visualizar el modo en que se han producido la inmersión virtual, y establecer un diálogo con el grupo a través de revivir sus experiencias mediadas, pero siempre en primera persona.

\subsection{Evaluación}

Una vez concluido el taller el material queda depositado en formato virtual, y se exhibe a los padres mediante una localización SLURL (Location-Based Linking in Second Life), hecho que conlleva a los visitantes a participar y conocer el medio desde su nuevo cuerpo ingrávido. Cabe señalar que en ningún caso los participantes se han registrado, ni controlado su avatar común sin una supervisión externa; el objetivo es conocer el mundo virtual, no la autonomía del sujeto en Second Life. Mientras que en otros talleres dirigidos a un público más adulto, los asistentes pueden mostrarse críticos en sus intervenciones, las edades que comprendían este taller sólo contemplaban Second Life como una herramienta de creación y entendimiento del espacio virtual.

Con respecto al conjunto de la actividad, la principal contribución en esta inmersión es la idea de combinar y compatibilizar los recursos tecnológicos con 
estrategias de creación tradicional. Compartir las obras finales en formato papel, y paralelamente comprender como estos mismos elementos pueden conferir un sentido complementario en su plano virtual. Otro aspecto a destacar recae en las sinergias desarrolladas en la aula, el escenario virtual como artífice de motivación creativa y como zona de interacción colectiva. El entorno virtual por otro lado ayuda a eliminar barreras físicas, pero también a unificar a sus integrantes en posiciones equidistantes; una proximidad que a su vez aprovecha el tutor para canalizar su imaginación y materializar su producción.

\section{Culdesac Island laboratorio plural}

La actividad aquí descrita forma parte de una gran variedad de talleres, mayoritariamente pensados para adultos, en los que sobre una misma isla virtual convergían proyectos e intenciones muy diversificados. Ya sea desde pases audiovisuales, generación de movimientos anatómicos, cursos virtuales de machinima, diseño 3D de objetos para humanizar el entorno, conferencias, performances en línea o actuaciones musicales en tiempo real. Con esta anotación, quiero manifestar el potencial de los mundos virtuales en su faceta educativa, así como su importancia en el conjunto de procesos creativos; un visión que acerca esta tecnología a un complemento de aquello real, y no a un estadio irreal. Por lo tanto, la misión de los artistas y educadores es dar a conocer estas plataformas desde su lado cultural, productivo y ético; en definitiva dar las herramientas necesarias para aprovechar su potencial en un formato pedagógico $^{11}$ y de comprensión de nuestro mundo global. Como nos apuntaba Ana Iribas (2007: 15): «Parece lógico contemplar la docencia en SL como un complemento a la presencial y a la del Campus Virtual habitual, y no como un sustituto. La manera en la que se implemente la enseñanza de las universidades españolas en Second Life está por definir».

La particularidad del taller expuesto tiene como eje sus "actores": los niños. El aprendizaje de forma participativa es el nexo que une los diferentes recorridos de las prácticas (creación de avatar, dérive, diseño, museu interactivo, machinima...). El mundo virtual nos proporciona un escenario pedagógico en el que el "juego", y sus transacciones simbólicas, entran en contacto con las posibilidades hipertextuales del metaverso, sus representaciones en un espacio tridimensional, y una vía alternativa para expandir las actividades de la real life. Mediante un nuevo sistema de comunicación, el mundo virtual, los alumnos exploran Second Life utilizando su creatividad para seguir avanzando entre símbolos; una forma de capturar nuevos registros y ampliar la cognición de lo virtual desde la acción, lo lúdico y lo metafórico. Por lo tanto, el resultado final no recae en desplazar las actividades docentes sobre un plano digital, sino en ramificar los contenidos sobre nuevos canales que permitan la obtención de nuevas metodologías para el desarrollo creativo. El reto de trabajar con niños responde a la voluntad de iniciar procesos experimentales a través de sus capacidades plásticas en combinación con una interfaz 3D, y visualizar como ésta puede suponer un estímulo en su construcción abstracta de la realidad. Quizás la clave de la futura enseñanza sea minimizar la distancia entre el espacio real y el metaverso, no simplemente como distintos canales de comunicación, sino como canales de desarrollo, cooperación e integración de conocimiento desde la interacción. 


\section{Referencias}

Barthes, R. (1970): S/Z, Seuil, Paris.

Castronova, E. (2001): «Virtual Worlds: A First-Hand Account of Market and Society on the Cyberian Frontier». En CESifo Working Paper No. 618.

Calongne, C.M. (2008, Sep/Oct.): «Educational Frontiers: Learning in a virtual World», Educase review, 43 (5). Fuente en línea: <http://www.educase.edu/ library/ erm0852> (Fecha de consulta: 03/02/2010)

Iribas, A. (2007): «Enseñanza virtual en Second Life: una opción online animada para las universidades y las artes», Dpto. De Pintura, Facultad de Bellas Artes, UCM.

Kelland, M.; Dave, M. (2005): Machinima, Thompson: Boston.

Young, J.; Jones, G., y Kalinowski, K. (2005): «Virtual Learning Environments Technology for Interactive Class Discussion». Comunicación en la 4th International Conference on Technology in Teaching and Learning in Higher Education, Pekín, China.

Wheeler, S.; Kelly, P. \& Gale, K. (2005): «The influence of online problem-based learning on teachers' professional practice styles», ALT-J: Research in Learning Technology, 13, 125-37.

\section{Notas}

1. Véase documentación en línea: <http://www.ciudades3-0.eu/> (Fecha consulta: $11 / 12 / 2012)$

2. Véase archivos en línea del proyecto estructurado bajo 23 cápsulas audiovisuales: $<\mathrm{http}$ :// vimeo.com/24621230> (Fecha consulta: 09/10/2012)

3. Véase portal oficial: $<$ http://secondlife.com/> (Fecha consulta: 14/08/2011)

4. El término metaverso se identifica con la idea de mundo paralelo, y proviene de la novela Snow Crash, publicada en 1992 por Neal Stephenson. También se utiliza habitualmente para describir la visión de trabajo en espacios 3D totalmente inmersivos.

5. Véase portal de intercambio de divisas entre la moneda virtual de Second Life (L\$) y su precio actual en el mercado: <http://www.xchange4ls.com/> (Fecha de consulta: $10 / 11 / 2011)$

6. Véase condiciones de uso: $<$ http://wiki.secondlife.com/wiki/Linden_Lab_Official:Teens _ in_Second_Life $>$ (Fecha consulta: 23/01/2012)

7. Peer to peer ( $22 \mathrm{p})$, "de igual a igual", es un término para designar un sistema de comunicación en red que no tiene ni servidores ni clientes fijos; son nodos que se comportan tanto como clientes como servidores de otros nodos. Véase: <http://www. ctheory.net/articles.aspx?id=499> (Fecha de consulta: 18/09/2008)

8. Véase comunidad machinima en Second Life: <http://slmachinimaarts.ning.com/> (Fecha de consulta: 12/02/2009)

9. Véase obra artística de la code performer Gazira Babeli: < http://gazirababeli.com/> (Fecha de consulta: 02/03/2009) 
10. El neologismo hyperformalism fue acuñado por el artista virtual Dan Coyote: $<$ http:// www.dancoyote.com> (Fecha de consulta: 15/10/2010)

11. Véase recursos educativos de Second Life: $<$ http://wiki.secondlife.com/wiki/Second Life_Education> (Fecha de consulta: 18/04/2011) 